\title{
Transient dynamic analysis of pile foundation responses due to ocean waves using the scaled boundary finite element method
}

\author{
Miao Li ${ }^{1,2} \cdot$ Hong Guan ${ }^{2} \cdot$ Hong Zhang ${ }^{2}$
}

Received: 14 October 2015 / Accepted: 22 February 2016 / Published online: 16 March 2016

(C) Springer International Publishing Switzerland 2016

\begin{abstract}
In this study, a semi-analytical numerical method, the scaled boundary finite element method, is employed to investigate the time-dependent behaviour of pile foundations when subjected to ocean wave loads. Individual piles present steady-state vibrations at the same frequency as external waves considering the material damping effect. In the case with a group of two piles, the structural behaviour of pile foundations is a multivariable function of the wave properties and the spatial arrangement of piles, and each pile responses differently to various parameter combinations. The leading pile, which interacts with incoming waves earlier, normally experiences greater displacement amplitude than the subsequent pile. Both piles show relatively greater displacement in cases with large wave numbers. When the two piles are closely spaced or when the wave number is small, their responses tend to have phase difference and move towards different directions. This study is expected to shed light on the transient behaviour of pile foundations, when wave load is a dominant factor.
\end{abstract}

Keywords Time-dependent responses - Pile foundations . Wave loads $\cdot$ Scaled boundary finite element method

Hong Zhang

hong.zhang@griffith.edu.au

1 Engineering, Faculty of Business, Charles Sturt University, Engineering Building 1305, Panorama Avenue, Bathurst, NSW 2795, Australia

2 Griffith School of Engineering, Griffith University, Gold coast, QLD 4222, Australia

\section{Introduction}

Investigations of engineering accidents in ocean environment revealed that waves are of paramount importance for the safe design and reliable utilisation of ocean installations (Benassai 2006; Gōda 2010; Moan 2005; United States Coast Guard 1984). This has led to a significant amount of studies and formulated the framework of wave-structure interaction (WSI). Over the past few decades, the study of WSI problems has developed from first-order linear waves (Feng et al. 2008; Finnegan and Goggins 2012; Li et al. 2011; Spring and Monkmeyer 1974) to high-order non-linear waves (Bai and Taylor 2006; Malenica et al. 1999; Wang and Wu 2007, 2010; Zhong and Wang 2009), and from simply configured structures (Chen et al. 2009; Tao et al. 2007; Zhu 1993) to groups of complex structures (Bai and Taylor 2009; Chatjigeorgiou and Mavrakos 2010; Li et al. 2013a, b; Liu et al. 2013; Song et al. 2010). However, most of the WSI studies focused on solving wave field data, for example free surface elevation and velocity field in the presence of structures (Ducrozet et al. 2010; Huang and Taylor 1996; Jung et al. 2004; Liu et al. 2013; Wu et al. 2013). The subsequent structural responses have received insufficient attention. In cases with complex structures where explicit wave expressions are not available, structural engineers face additional challenges to formulate the linkage between wave forces and structural responses.

Concerning the complexity involved in WSI problems, it is practically difficult to seek analytical or experimental solutions to address structural responses under wave loads. A few reported numerical studies set a precedent in this regard. $\mathrm{Wu}$ et al. (1995) investigated the wave-induced response of an elastic floating plate. Lee and Wang (2000) addressed the dynamic behaviour of a tension leg platform with net-cage system when exposed to ocean waves. Srisupattarawanit et al. (2006) presented a time-accurate computation of the interac- 
tion between elastic structures and random waves of a finite water depth. Ge et al. (2010) investigated the interaction between waves and a submerged floating tunnel. However, there are limitations associated with the abovementioned studies, which are directly or indirectly related to the numerical methods employed in the analyses. The popular and widely used finite element method (FEM) is very flexible in dealing with inhomogeneous and anisotropic structures, but runs into difficulties when extensive computational domains are involved. The boundary element method (BEM) is an attractive alternative to FEM when modelling unbounded domains, i.e. the wave field in this circumstance. Nevertheless, the complexity of the fundamental solution increases dramatically with the complexity of the physical problem.

A semi-analytical numerical method developed by Wolf and Song (1996), termed as the scaled boundary finite element method (SBFEM), overcomes the abovementioned disadvantages of FEM and BEM. It possesses favourable features by combining the following advantages: significantly releasing the computational burden of three-dimensional calculations; precisely satisfying boundary conditions at infinity; not requiring fundamental solutions and being free from irregular frequencies and singular integrals. SBFEM has been employed in various engineering fields with rapid recognition (Li 2007; Tao and Song 2008; Tao et al. 2009a, b). The authors employed SBFEM to investigate the structural response of offshore monopiles subjected to wave loads (Li et al. 2010, 2011) and subsequently developed a threedimensional SBFEM model to address wave field behaviour in the presence of structures and the structural response due to wave loads ( $\mathrm{Li}$ et al. 2013a, b). These studies were conducted in a static scope without considering the time factor. The current analysis further advances the SBFEM model to the time-dependent context to capture the transient dynamic responses of structures when subjected to ocean wave loads. This study is believed to offer more realistic information in the foundation design processes for ocean constructions.

\section{Problem formulation}

A group of pile foundations engaged in an ocean environment is used to illustrate the physical problem, as shown in Fig. 1. In the study of WSI problems, important progress has been made in incorporating seabed effects. Mostafa and El Naggar (2004) considered soil-structure interaction when studying offshore platforms, representing the $p-y$ (net reaction on pile per unit length - pile lateral deflection) curve of the pile foundations in the real situation. Jeng et al. (2013); Ye et al. (2013a,b) developed and validated two-dimensional and/or three-dimensional coupled and/or semi-coupled numerical models and addressed wave and porous seabed domains. Ye et al. $(2014,2015)$ examined the dynamic responses of com-

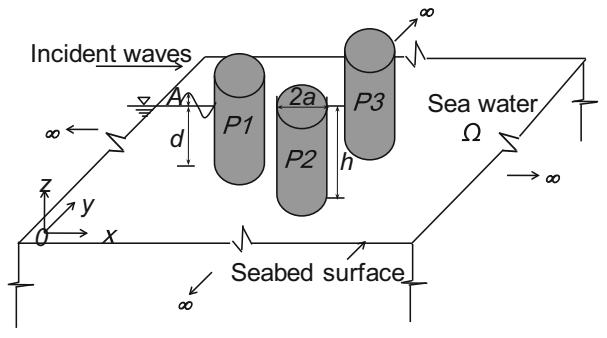

Fig. 1 A group of pile foundations in ocean environment (reproduced from Li et al. 2013b)

posite breakwaters in the scope of fluid-structure-seabed interaction. These studies offer a good guidance as to how the present analysis could be further developed. As an initial attempt to formulate an SBFEM solution to the proposed physical problem, pile foundations are assumed to be fixed at the seabed level in this study. Relative motions between pile foundations and the seabed are not considered. In addition, as the main focus of this study lies in the behaviour of pile foundations in response to ocean wave loads, external loads from winds acting on wind farm monopiles or superstructures on pile group foundations are not considered. In Fig. 1, the origin $O$ of the Cartesian coordinate system is located at the seabed surface. $x$ and $y$ denote two orthogonal horizontal directions, and $z$ positively points upwards. Here, $\Omega$ represents the three-dimensional ocean field; $a$ and $h$ stand for the pile radius and pile height, respectively; $A$ symbolises the wave amplitude, and $d$ the water depth.

\subsection{Wave behaviour}

Though quite a few studies have been directed to highorder non-linear waves, the formulation presented in this study is limited to the linear scope. The linear wave theory offers a description of wave parameters with sufficient accuracy for many purposes (Dean and Dalrymple 1991; Gōda 2010), such as the structural design and reliability evaluation from the engineering point of view. Moreover, linear theory allows for a quick and preliminary evaluation of wave properties and their effects on structures. In addition, non-linear kinematic and dynamic wave parameters can be estimated from linear analysis results (Phillips 1977). Therefore, it is conducive to linearly formulate SBFEM to solve complex interaction problems as an initial trial. In these circumstances, the seawater is assumed inviscid and incompressible, and the wave motion is irrotational. The velocity potential $\Phi$ of the three-dimensional wave field $\Omega$ is governed by the Laplace equation (Mei 1989):

$\nabla^{2} \Phi=0$ in $\Omega$

The concept of separation of variables is employed to decompose $\Phi$ into univariate functions with respect to independent 
variables, i.e. the spatial variables $x, y$, and $z$, and the temporal variable $t$ :

$\Phi(x, y, z, t)=\phi(x, y) Z(z) e^{-i \omega t}$

In Eq. (2), $i=\sqrt{-1}$; $\omega$ is the angular frequency; $\phi(x, y)$ is governed by the Helmholtz equation at the free surface level (Mei 1989):

$\nabla^{2} \phi+k^{2} \phi=0 \quad(k:$ the wave number $)$

Normally, the geometric dimension of pile foundations is incomparable with the wavelength. Hence, the total velocity potential of the wave filed (denoted by a subscript ' $T$ ') is formulated by the summation of the velocity potentials of the incident (denoted by a subscript ' $I$ ') and the scatter wave (denoted by a subscript ' $S$ '). Based on the superposition principle, $\Phi$ and $\phi$ in Eqs. (1), (2) and (3) represent any of the total, the incident and the scattered velocity potentials. For ease of discussion, a radial direction $r$ and the corresponding tangential direction $n$ are used when introducing the boundary conditions associated with the Helmholtz equation (3). At the wetted structural surface, the Neumann boundary condition is enforced as (for concise presentation, the comma notation for partial derivatives is used):

$\phi_{T, n}=0$

At infinity, the Sommerfeld radiation condition (Sommerfeld 1949 ) is specified in terms of the scattered waves as:

$\lim _{k r \rightarrow \infty}(k r)^{1 / 2}\left(\phi_{S, r}-i k \phi_{S}\right)=0$

Once $\phi$ in Eq. (3) is solved with the boundary conditions specified in Eqs. (4) and (5), the free surface elevation $\eta_{\theta}$ can be calculated using the gravitational acceleration $g$ as:

$\eta_{\theta}=\frac{i \omega}{g} \phi e^{-i \omega t}$

In Eq. (2), the function $Z(z)$ is analytically formulated as:

$Z(z)=\frac{\cosh k z^{\prime}}{\cosh k d}$

with the stretched coordinate $z^{\prime}$ calculated according to Wheeler (1969) as:

$z^{\prime}=\frac{z d}{d+\eta_{\theta}}$

Subsequently, the dynamic wave pressure $p_{d}$ acting on pile foundations can be computed as:

$p_{d}=-\rho_{w} \Phi,{ }_{t}$ with $\rho_{w}$ being the density of the sea water. The total wave pressure acting on pile foundations results from the superposition of the dynamic pressure $p_{d}$ and the hydrostatic pressure $p_{h}$, which is formulated as:

$p_{h}=\rho_{w} g\left(d-z^{\prime}\right)$

\subsection{Structural response}

The structural behaviour of pile foundations is governed by the elasto-dynamic differential equations:

$$
\begin{aligned}
& {[L]^{T}\{\sigma\}+\omega^{2} \rho_{p}\{u\}=0} \\
& \{\sigma\}=[D]\{\varepsilon\} \\
& \{\varepsilon\}=[L]\{u\}
\end{aligned}
$$

where $[L]$ is the partial differential operator; $\{u\},\{\sigma\}$ and $\{\varepsilon\}$ represent the pile displacement, stress and strain amplitudes, respectively; $[D]$ is the elastic matrix, and $\rho_{p}$ denotes the mass density of pile foundations. Equation (11) is solved with the boundary condition specified at the seabed level where the displacement of pile foundations is assumed as zero, i.e.

$\{u\}=0$, at $z=0$.

and the piles are subjected to the time-dependent wave force summed from the dynamic and hydrostatic components:

$p_{w}=p_{d}+p_{h}$

\section{SBFEM model}

A three-dimensional SBFEM model addressing wave-structure interaction problems in the static scope has been developed and systematically documented by Li et al. (2013a,b). In the present study, the SBFEM formulation of the wave field remains the same. The formulation of pile foundations and the corresponding solution procedure, on the other hand, differ greatly from those specified in the static analysis and require more advanced techniques.

The SBFEM equation expressed in nodal displacement $\{u(\xi)\}$ corresponding to Eq. (11) is written as:

$$
\begin{array}{r}
{\left[E^{0}\right] \xi^{2}\{u(\xi)\}_{, \xi \xi}+\left(2\left[E^{0}\right]+\left[E^{1}\right]^{T}-\left[E^{1}\right]\right) \xi\{u(\xi)\}_{, \xi}} \\
+\left(\left[E^{1}\right]^{T}-\left[E^{2}\right]\right)\{u(\xi)\}+\omega^{2}\left[M^{0}\right] \xi^{2}\{u(\xi)\}=0
\end{array}
$$

with $\left[E^{0}\right],\left[E^{1}\right],\left[E^{2}\right]$ and $\left[M^{0}\right]$ being coefficient matrices calculated from the scaled boundary discretisation.

Using the dynamic stiffness matrix $[S(\omega)]$, Eq. (14) can be rewritten as: 


$$
\begin{aligned}
& \left([S(\omega)]-\left[E^{1}\right]\right)\left[E^{0}\right]^{-1}\left([S(\omega)]-\left[E^{1}\right]^{T}\right)-\left[E^{2}\right] \\
& \quad+[S(\omega)]+\omega[S(\omega)]_{, \omega}+\omega^{2}\left[M^{0}\right]=0
\end{aligned}
$$

$[S(\omega)]$ is to be solved from Eq. (15) and substituted into the corresponding nodal force $\{R\}$-nodal displacement $\{u\}$ relationship formulated on the discretised boundary:

$$
\{R\}=[S(\omega)]\{u\}
$$

Equation (15) is a non-linear first-order matrix-form Riccati differential equation. Solving the dynamic stiffness matrix $[S(\omega)]$ from it is not a straightforward task. Song (2009) employed the continued fraction technique to postulate the formation of the dynamic stiffness matrix, using the stiffness matrix $[K]$ and the mass matrix $[M]$ as:

$$
\begin{aligned}
& {[S(x)]} \\
& \quad=[K]+x[M]-x^{2}\left[S^{(1)}(x)\right]^{-1}=[K]+x[M] \\
& \quad-\frac{x^{2}}{S_{0}^{(1)}(x)+x S_{1}^{(1)}(x)-\frac{x^{2}}{S_{0}^{(2)}(x)+x S_{1}^{(2)}(x)-\ldots \frac{x^{2}\left(M_{c f}\right)(x)+x S_{1}^{(M)}(x)}{\left.S_{c f}\right)}}}
\end{aligned}
$$

with $x=-\omega^{2}$ and $M_{c f}$ represents the order of the continued fraction. The advantages of using continued fractions in SBFEM lie in that, for low frequency excitations, structural behaviour can be represented by the stiffness and mass matrices ([K] and $[M])$ in Eq. (17). A zero or low order of $M_{c f}$ can be assigned to retain the original computational expenses in the analysis. For high frequency applications, the dynamic properties of the structure can be described using high-order continued fraction terms. No mesh refinement is required, as would be the case in an FEM analysis. Key solution procedures in Song and Tao (2009) are followed herein for integrity of presentation. Substituting Eq. (17) into Eq. (16) yields the following expression:

$$
\{R\}=[K]\{u\}+x[M]\{u\}-x\left\{u^{(1)}\right\}
$$

with

$$
x\left\{u^{(1)}\right\}=x^{2}\left[S^{(1)}(x)\right]^{-1}\{u\}
$$

Equation (19) can be reformulated as:

$$
x\{u\}=\left[S^{(1)}(x)\right]\left\{u^{(1)}\right\}
$$

$\left[S^{(1)}(x)\right]$ and Eq. (20) are generalised for the $i$ th order continued fraction as:

$$
\left[S^{(i)}(x)\right]=\left[S_{0}^{(i)}\right]+x\left[S_{1}^{(i)}\right]-x^{2}\left[S^{(i+1)}(x)\right]^{-1}
$$

and

$x\left\{u^{(i-1)}\right\}=\left[S^{(i)}(x)\right]\left\{u^{(i)}\right\}(i \geq 1)$

where $\left\{u^{(i)}\right\}(i \geq 1)$ are the auxiliary displacements introduced by the order of the continued fraction. Substituting Eq. (21) into Eq. (22) and using Eq. (19) lead to:

$$
\begin{aligned}
x\left\{u^{(i-1)}\right\}= & {\left[S_{0}^{(i)}\right]\left\{u^{(i)}\right\}+x\left[S_{1}^{(i)}\right]\left\{u^{(i)}\right\} } \\
& -x\left\{u^{(i+1)}\right\}(i \geq 1)
\end{aligned}
$$

Rearrange Eqs. (18) and (23) into similar form as:

$$
\begin{aligned}
& {[K]\{u\}+x[M]\{u\}-x[I]\left\{u^{(1)}\right\}=\{R\}} \\
& {\left[S_{0}^{(i)}\right]\left\{u^{(i)}\right\}-x[I]\left\{u^{(i-1)}\right\}} \\
& \quad+x\left[S_{1}^{(i)}\right]\left\{u^{(i)}\right\}-x[I]\left\{u^{(i+1)}\right\}=0
\end{aligned}
$$

Note that the first equation in Eq. (24) corresponds to $i=$ 0 and $\{u\}$ represents the structural DOFs. The second equation corresponds to $i \geq 1$ and $\left\{u^{(i)}\right\}$ stands for the introduced auxiliary DOFs from the continued fraction. These two equations can be combined and written in matrix-form algebraic equations as:

$$
\left(\left[K_{h}\right]-\omega^{2}\left[M_{h}\right]\right)\{y\}=\{f\}
$$

with

$\left[K_{h}\right]=\operatorname{diag}\left([K]\left[S_{0}^{(1)}\right]\left[S_{0}^{(2)}\right] \cdots\left[S_{0}^{\left(M_{c f}\right)}\right]\right)$

$\left[M_{h}\right]=\left[\begin{array}{lllll}{[M]} & -[I] & 0 & \cdots & 0 \\ -[I] & {\left[S_{1}^{(1)}\right]} & -[I] & \cdots & 0 \\ 0 & -[I] & {\left[S_{1}^{(2)}\right]} & \cdots & 0 \\ \vdots & \vdots & \vdots & \ddots & \vdots \\ 0 & 0 & 0 & \cdots & {\left[S_{1}^{\left(M_{c f}\right)}\right]}\end{array}\right]$

$\{y\}=\left\{\{u\}\left\{u^{(1)}\right\}\left\{u^{(2)}\right\} \cdots\left\{u^{\left(M_{c f}\right)}\right\}\right\}^{T}$

and

$\{f\}=\left\{\begin{array}{llllll}\{R & 0 & 0 & \cdots & 0\end{array}\right\}^{T}$

By the inverse Fourier transformation, Eq. (25) is rewritten in the time domain as:

$\left[K_{h}\right]\{y\}+\left[M_{h}\right]\{\ddot{y}\}=\{f\}$ 
The coefficient matrices $\left[K_{h}\right]$ and $\left[M_{h}\right]$ listed in Eqs. (26) and (27) are calculated for each subdomain. They are assembled into global matrices $\left[K_{G}\right]$ and $\left[M_{G}\right]$ in such a way that the entries corresponding to structural DOFs follow the assemblage concept in FEM, and those corresponding to the auxiliary DOFs are allocated into the global matrices in sequence.

$$
\left[K_{G}\right]\{y\}+\left[M_{G}\right]\{\ddot{y}\}=\{f\}
$$

It is worth mentioning that the number of DOFs involved in the assembled global equation of motion (Eq. 31) is larger than the number of structural DOFs due to the use of the continued fraction technique. The higher the order of continued fraction, the greater the number of DOFs and accordingly the more intensive the computational demand. An appropriate continued fraction order should be considered to avoid the computational memory issue. Equation (31) represents an undamped forced vibration. It can be employed to examine natural frequencies of structures if the external excitation $\{f\}$ equals 0 . In this study, the material damping effect is considered by adding the damping force into Eq. (31), which leads to (the subscript ' $G$ ' is dropped for conciseness):

$$
[M]\{\ddot{y}\}+[C]\{\dot{y}\}+[K]\{y\}=\{f\}
$$

with $[C]$ being the damping matrix and formulated by the linear combination of the stiffness matrix $[K]$ and the mass matrix $[M]$ as:

$[C]=\alpha_{d}[M]+\beta_{d}[K]$

$\alpha_{d}$ and $\beta_{d}$ are two frequency-independent coefficients. They are calculated from:

$$
\begin{aligned}
\alpha_{d} & =2 r_{m d} \omega_{1} \omega_{2} /\left(\omega_{1}+\omega_{2}\right) \\
\beta_{d} & =2 r_{m d} /\left(\omega_{1}+\omega_{2}\right)
\end{aligned}
$$

where $r_{m d}$ is the material damping ratio. $\omega_{1}$ and $\omega_{2}$ are natural circular frequencies corresponding to two orthogonal modal shapes of the structure.

Equation (32) is solved in the time domain using the Newmark integral technique. Once the time history of the nodal displacement is obtained, the time-dependent behaviour of the pile foundation is sought. A detailed solution procedure incorporating both the wave and structural analyses is illustrated in Fig. 2.

\section{Model validation}

The validation of the SBFEM model is conducted in two aspects, i.e., wave and structural analyses. A validation case in terms of the wave analysis can be found in ( $\mathrm{Li}$ et al. 2013b), in which the free surface elevation around pile foundation circumference calculated from the SBFEM model was compared against an analytical expression. To further justify the applicability of the proposed model and demonstrate the validity of the linear wave theory, the experiment conducted by Chakrabarti and Tam (1975) is reproduced. In Chakrabarti and Tam's experiment, dynamic wave effects on a large vertical cylinder were measured in a wave tank in the form of pressure, force and moment. Detailed information about the experimental model, apparatus and the test procedure can be found in Chakrabarti and Tam (1975). Results from the experiment generated a plot of the effective inertia coefficient $C_{M}$ versus the ratio of the cylinder diameter to wavelength $k a / \pi$, as shown in Fig. 3. Corresponding SBFEM results using the proposed model are also plotted. The agreement between the modelling and the experimental results demonstrated the credibility of the SBFEM model in solving wave domain.

In terms of the validation of the SBFEM formulation for structural analysis, the $L$-shaped panel discussed in Section 6.2 in Song (2009) is analysed herein. The $L$-shaped panel is subjected to a triangular impulse as shown in Fig. 4. Relevant information of the validation case is specified in Table 1. Using the proposed SBFEM model, both the natural frequencies of the $L$-shaped panel and the vertical displacements of Points $A$ and $B$ are presented. The results are compared against those from an equivalent and converged finite element analysis. In the SBFEM model, three subdomains (super element) are designed as shown in Fig. 5. In each subdomain, the scaling centre is located at the geometric centre of the domain. Fifty 3-node quadratic elements, resulting in a total of 98 nodes, are used to discretise the boundaries and subdomain interfaces of the panel. As shown in Fig. 6, a parametric analysis on the number of continued fractions, with $M_{c f}=0,1,2,3$ and 4, is conducted based on the above mesh configuration. It is observed that the result converges rapidly as $M_{c f}$ increases from 0 to 3 . Both the natural frequencies calculated from $M_{c f}=3$ and 4 coincide well with the converged FEM results, as seen in the plot of relative errors in Fig. $6 \mathrm{~b}$. This indicates an order of $M_{c f}=3$ or 4 is sufficient for the specified mesh resolution.

An estimate of the optimum $M_{c f}$ prior to the calculation can be conducted according to Song (2009). The current meshing scheme discretises each segment $b$ with five 3 -node quadratic elements, leading to 11 nodes per length $b$. Considering the common rule of using six nodes per wavelength (Marburg 2002), the current meshing scheme is capable of modelling $11 / 6=1.83$ wavelengths per length $b$. The distance between the scaling centre and a vertex in a subdomain is $0.707 b$, which is equivalent to $0.707 \times 1.83=1.29$ wavelengths. Based on the guideline of three to four terms of continued fractions per wavelength (Song 2009), an order of 


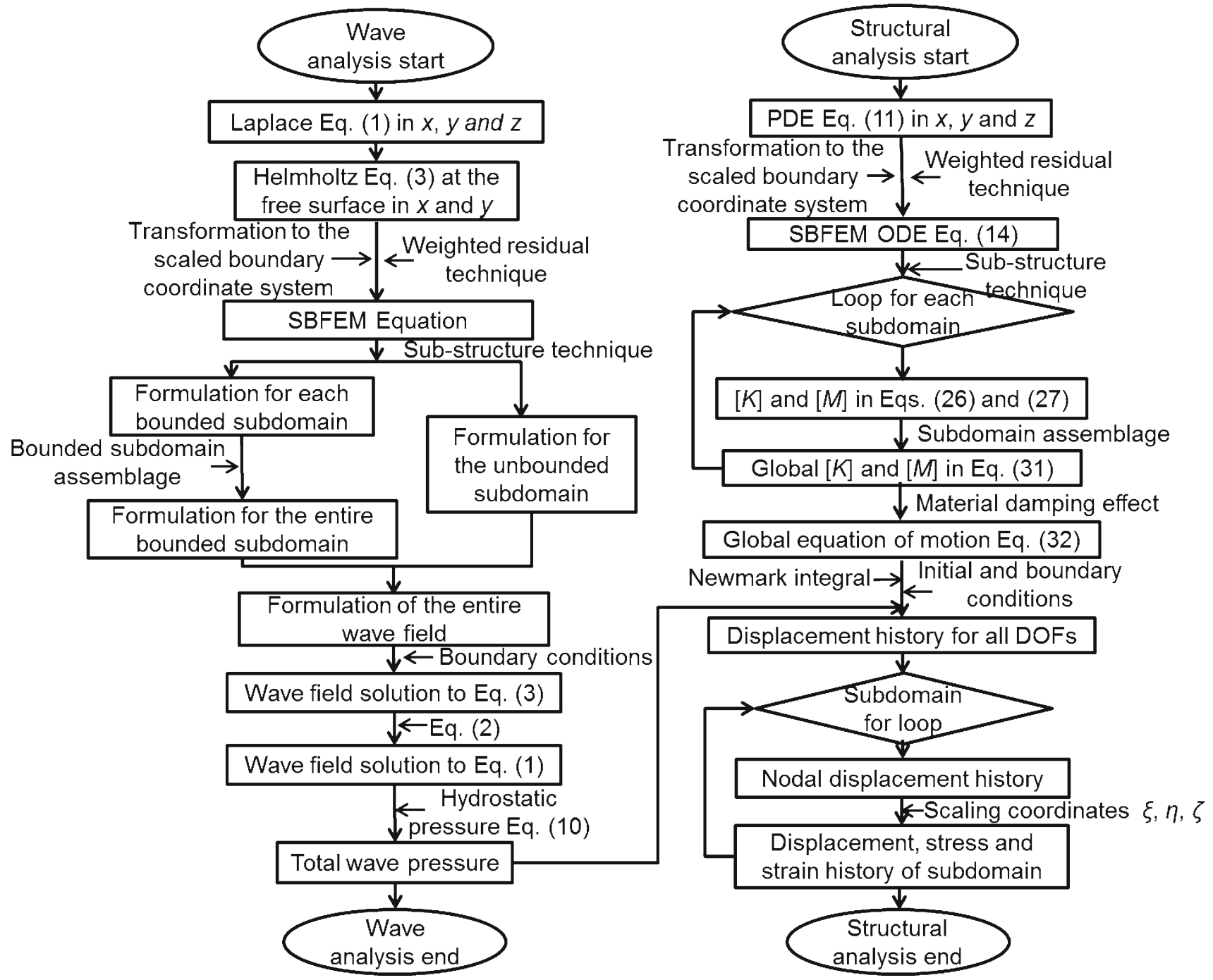

Fig. 2 Solution procedure of wave field and time-dependent structural analysis

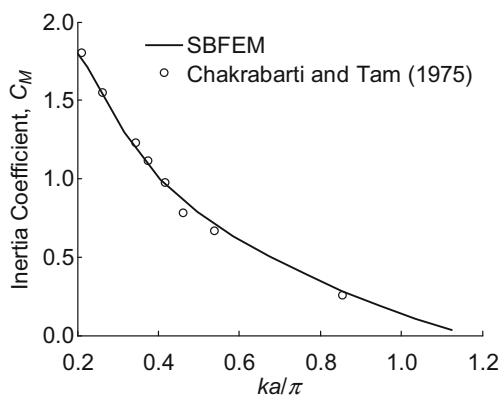

Fig. 3 Comparison between SBFEM model and experimental data for wave analysis

$M_{c f}=3$ or 4 is expected for accurate results. This corresponds well to the result of the parametric analysis. Figures 6 and 7 demonstrate a satisfactory level of accuracy and reliability of the SBFEM calculation. It worth mentioning that the equivalent converged FEM analysis requires 300 twodimensional 8-node quadratic solid elements, i.e., 981 nodes in the calculation.

\section{Time-dependent response of monopile foundations}

Before investigating the dynamic behaviour of individual pile foundations, it is necessary to conduct a modal analysis to examine the natural frequencies of the pile foundation in relation to the excitation frequencies from external waves. Employing the parameters in Li et al. (2013b), a monopile foundation with its geometric configuration and material information listed in Table 2 is illustrated in Fig. 8. In the modal analysis, an undamped free vibration is examined by solving Eq. (31) with vector $\{f\}$ replaced by 0 . The first two 
Fig. 4 Model verification of the time-dependent structural analysis: a geometric configuration of an $L$-shaped panel (reproduced from Song 2009); b triangular impulse: variation of pressure $p$ versus time $t$

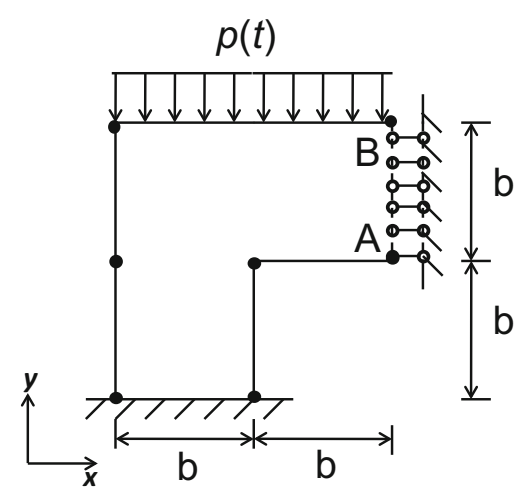

(a)

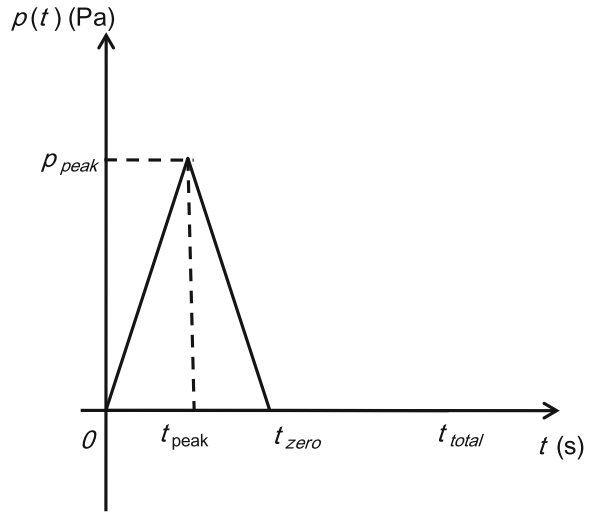

(b)
Table 1 Parametric information of SBFEM model validation for structural analysis

\begin{tabular}{llll}
\hline Parameters & Notations & Magnitudes & Units \\
\hline$L$-shaped panel & & & \\
Characteristic length & $b$ & 1 & $\mathrm{~m}$ \\
Young's modulus & $E$ & $2.8 \times 10^{10}$ & $\mathrm{~Pa}$ \\
Mass density & $\rho$ & 2400 & $\mathrm{~kg} / \mathrm{m}^{3}$ \\
Poisson's ratio & $v$ & $1 / 3$ & - \\
Temporal variables & $t_{\text {total }}$ & 0.022 & $\mathrm{~s}$ \\
& $t_{\text {peak }}$ & $1.46 \times 10^{-4}$ & $\mathrm{~s}$ \\
& $t_{\text {zero }}$ & $2.93 \times 10^{-4}$ & $\mathrm{~s}$ \\
& $\Delta t$ & $7.32 \times 10^{-6}$ & $\mathrm{~s}$ \\
Natural circular frequencies & $\omega_{1}$ & 1371.68 & $\mathrm{rad} / \mathrm{s}$ \\
& $\omega_{2}$ & 2819.27 & $\mathrm{rad} / \mathrm{s}$ \\
External force & $p_{\text {peak }}$ & $2.8 \times 10^{7}$ & $\mathrm{~Pa}$ \\
\hline
\end{tabular}

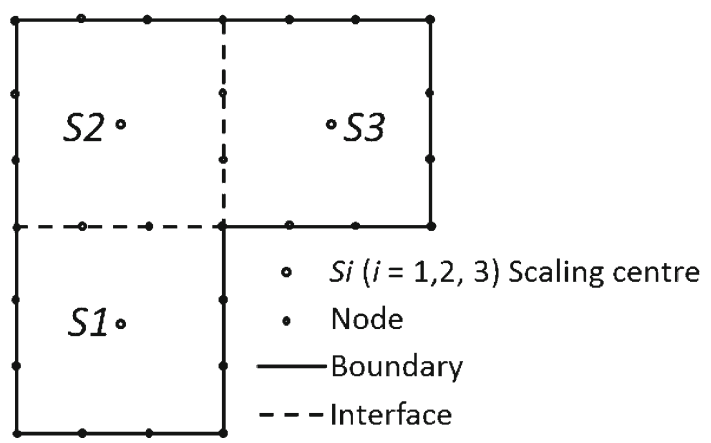

Fig. 5 SBFEM model of the $L$-shaped panel (reproduced from Li et al. 2015)

orders of the natural frequencies associated with the specified monopile foundation are $\omega_{1}=\omega_{2}=29.97 \mathrm{rad} / \mathrm{s}$. They are far greater than the excitation frequency of external waves, which is calculated to vary from 0.314 to $1.257 \mathrm{rad} / \mathrm{s}$ when the wave period in the normal ocean environment ranges between 5 and $20 \mathrm{~s}$. This will help to reduce the chance of resonance.
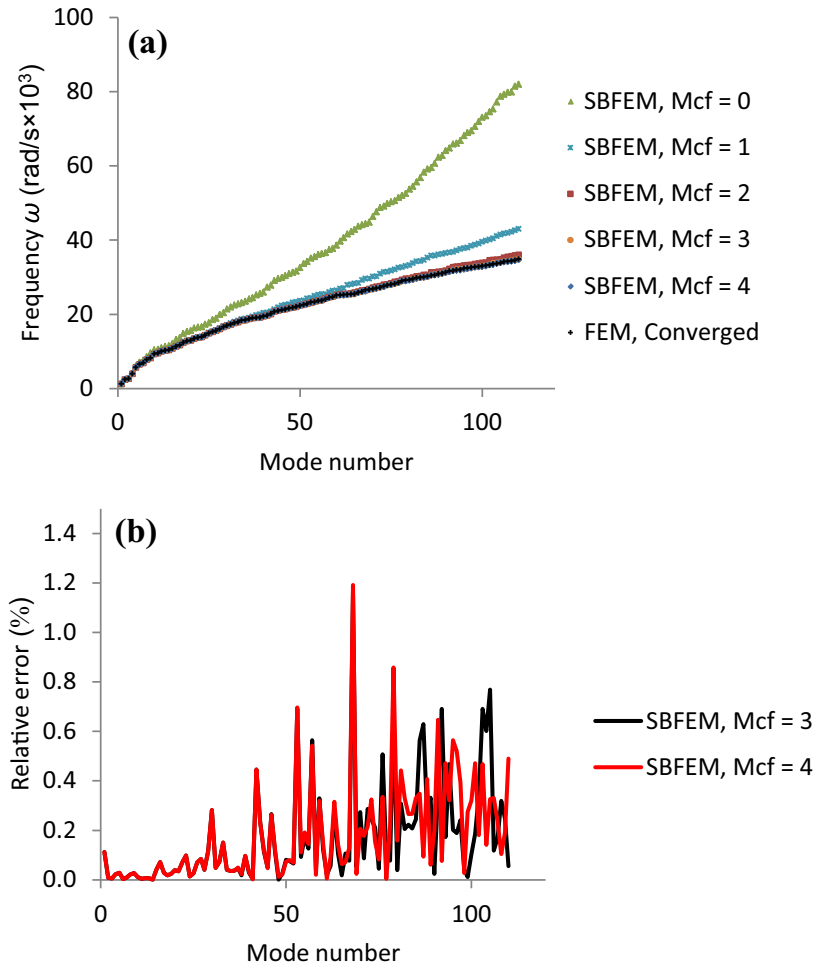

Fig. 6 Parametric analysis on the order of continued fractions and the comparison with the FEM results: a natural frequencies of the $L$-shaped panel and $\mathbf{b}$ relative error

In addition, the calculated natural frequencies $\omega_{1}$ and $\omega_{2}$ are to be used later to formulate the damping matrix $[C]$ in Eq. (33) when calculating the damped transient response of pile foundations subject to cyclic wave loads.

The transient response of Point $O^{\prime}$ (see Fig. 8) to the dynamic wave loads is illustrated in Fig. 9. The time variable $t$ is assumed to be zero at the instant the pile starts to withstand wave loads. A total of 50 periods of the pile motion are calculated and plotted. Employing the parameters listed in Table 2, it takes approximately ten periods for 
Fig. 7 Comparison of vertical displacement history of the $L$-shaped panel between SBFEM and FEM calculations at: a point $A$ and $\mathbf{b}$ point $B$

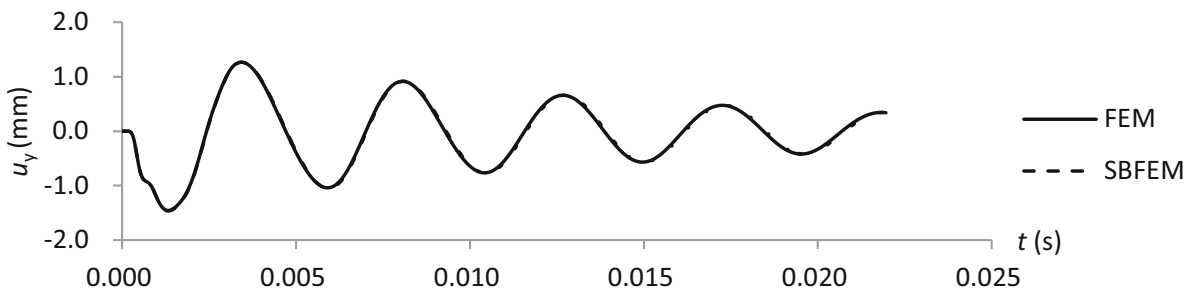

(a)

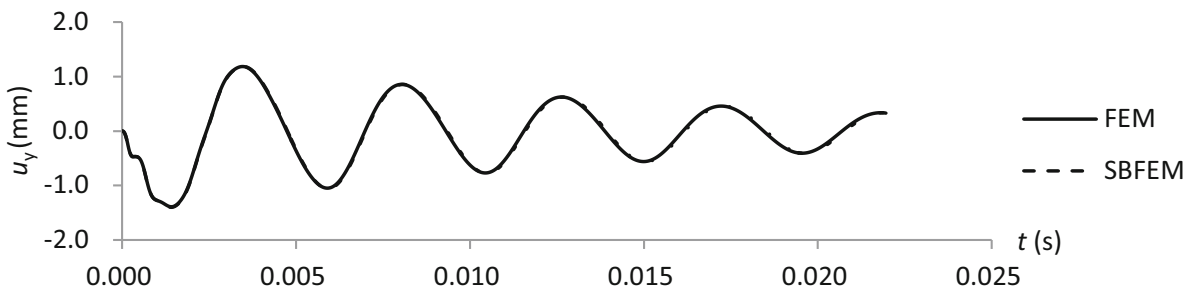

(b)
Table 2 Parameters of time-dependent analysis of a monopile foundation subjected to wave loads

\begin{tabular}{|c|c|c|c|}
\hline Parameters & Notations & Magnitudes & Units \\
\hline \multicolumn{4}{|l|}{ Pile parameters } \\
\hline Pile radius & $a$ & 0.5 & $\mathrm{~m}$ \\
\hline Pile height & $h$ & 10 & $\mathrm{~m}$ \\
\hline Young's modulus & $E$ & $2.8 \times 10^{10}$ & $\mathrm{~Pa}$ \\
\hline Mass density & $\rho_{p}$ & 2400 & $\mathrm{~kg} / \mathrm{m}^{3}$ \\
\hline Damping ratio & $r_{m d}$ & 0.05 & - \\
\hline Poisson's ratio & $v$ & 0.25 & - \\
\hline \multicolumn{4}{|l|}{ Wave parameters } \\
\hline Water depth & $d$ & 7.5 & $\mathrm{~m}$ \\
\hline Incident wave angle & $\alpha$ & 0 & $\mathrm{rad}$ \\
\hline Wave number & $k$ & 0.10 & $\mathrm{~m}^{-1}$ \\
\hline Water density & $\rho_{w}$ & 1000 & $\mathrm{~kg} / \mathrm{m}^{3}$ \\
\hline Wave amplitude & $A$ & 0.5 & $\mathrm{~m}$ \\
\hline \multicolumn{4}{|l|}{ Temporal variables } \\
\hline Time steps & $N$ & 800 & - \\
\hline Time interval & $\Delta t$ & 0.50 & $\mathrm{~s}$ \\
\hline Gravitational acceleration & $g$ & 9.81 & $\mathrm{~m} / \mathrm{s}^{2}$ \\
\hline
\end{tabular}

Part of the parametric values in Table 2 are sourced from Li et al. (2013b)

the mechanical energy associated with the pile's free motion to be dissipated. The pile thereafter arrives at a steady-state vibration and moves at the same frequency as waves. The following discussions are focused on the steady-state vibration considering the real ocean situation.

Figure 10a plots the displacement of two periods for five observation points located at different height levels along $L-L^{\prime}$, i.e. $z=2,4,6,8$ and $10 \mathrm{~m}$ (see Fig. 8a). It is observed that the five points move at the same frequency with different amplitudes, and the amplitude is greater at points further away from the seabed level. This feature can be more visually recognised in Fig. 10b, in which the motion of the pile for one complete period, represented by the movement of $L-L^{\prime}$, is depicted. The four curves labelled as 1,2, 3 and 4 match the four time instants marked by corresponding numbers in Fig. 10a. The pile starts off from its equilibrium position, denoted by curve 1, moves towards positive $x$ direction and reaches its maximum coverage marked by curve 2 at $t=T / 4$ (Here and onwards, $T$ denotes the period of the pile motion), then moves back to curve 3 and continues to swing to curve 4 to achieve the maximum on the other side, and eventually returns to its initial status upon completion of a cycle when $t=T$. The pile swings back and forth between curve 2 and curve 4 under the action of wave loads. Curve 1 or 3 denotes the equilibrium position of the pile in its cyclic movement. It does not coincide exactly with the pile's neutral still status due to the effect of the hydrostatic pressure, which accounts for the negative displacement appearing at the bottom of the pile. The hydrostatic pressure acts normally to the pile circumference and reaches its maximum at the seabed level as it linearly increases with the water depth.

Figure 11 reflects the displacements of five specific locations at the pile head level. Four of them are located along the pile circumference at $\theta=0, \pi / 2, \pi$ and $-\pi / 2$, and the fifth is selected at Point $O^{\prime}$, as shown in Fig. 8b. They present exactly the same motion pattern with identical frequency and amplitude. Based on the findings from Figs. 10b and 11, the subsequent discussions on the time-dependent response of pile foundations will be made by examining the dynamic behaviour of Point $O^{\prime}$ only. 
Fig. 8 A monopile foundation subjected to wave action a $x z$ plane view and $\mathbf{b} x y$ plane view of the pile head

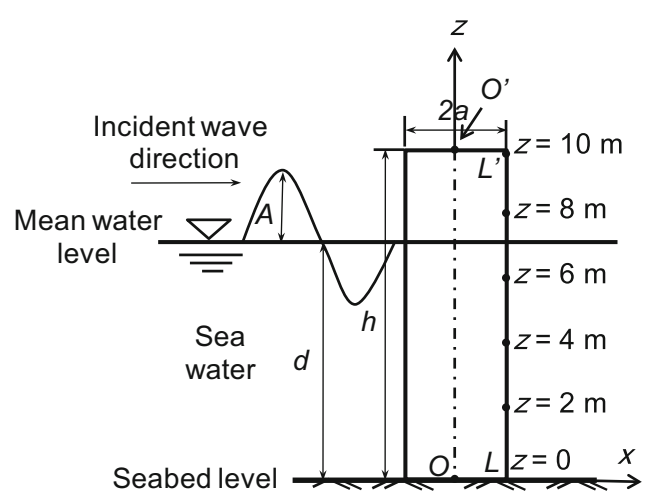

(a)

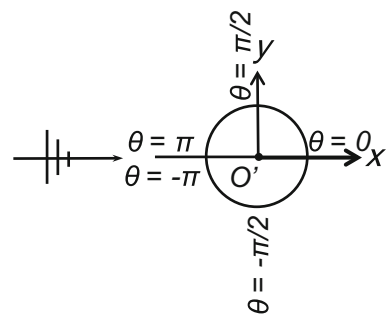

(b)

Fig. 9 Transient response of Point $O^{\prime}$ to the dynamic wave loads $\mathbf{a}$ in the $x$ direction and $\mathbf{b}$ in the $y$ direction

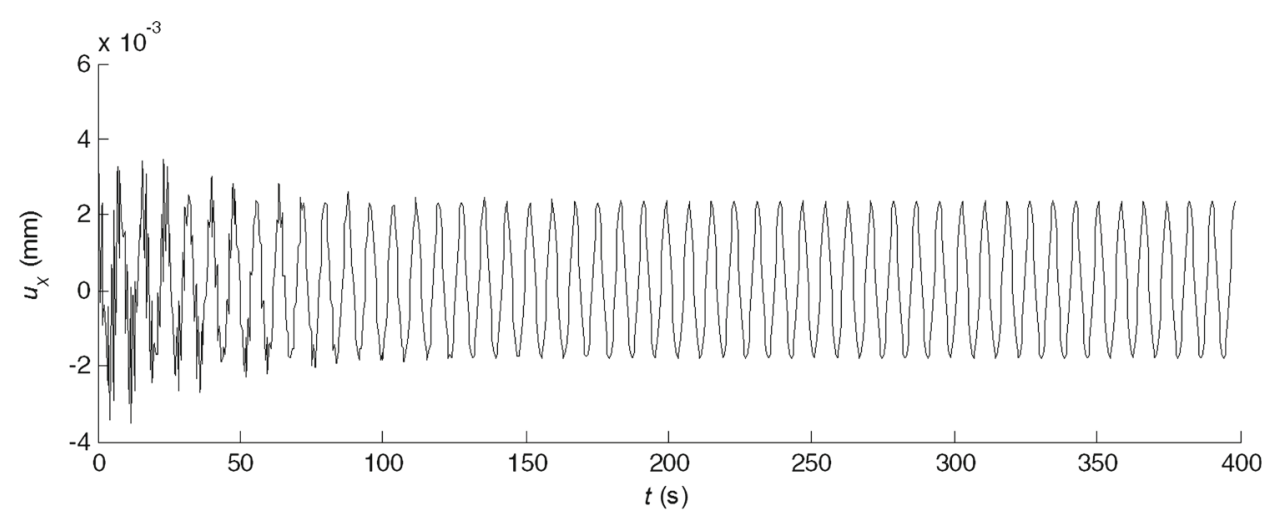

(a)

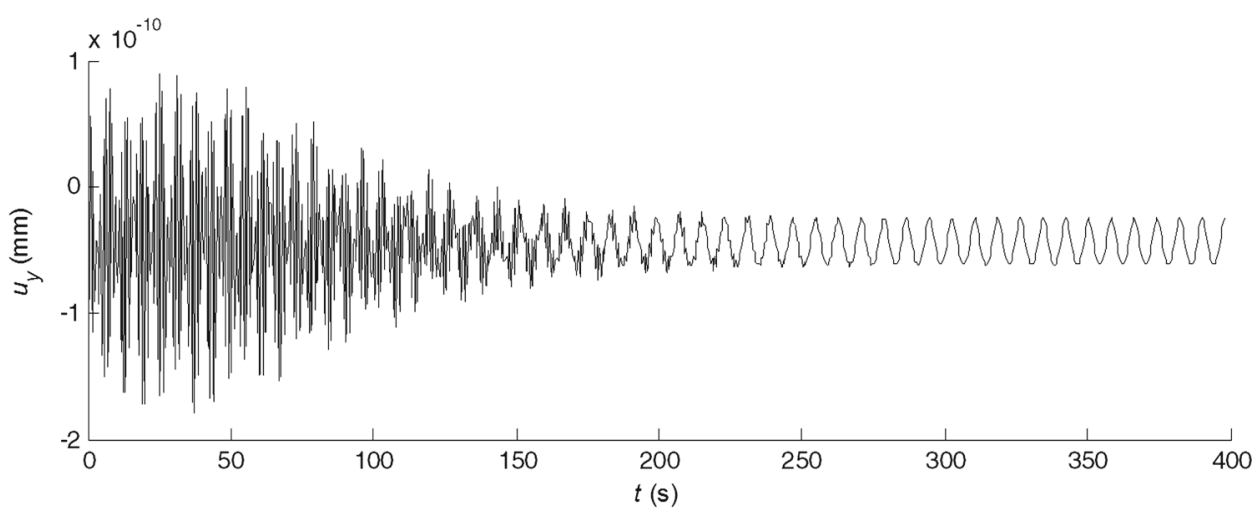

(b)

\section{Time-dependent response of pile group foundations}

The time-dependent response of a group of two piles is considered, with the intention to obtain enlightening information when more piles or complex structures are involved. The geometric sketch illustrating the physical problem is shown by an $x z$ plane view and an $x y$ plane view in Fig. 12. The two piles, denoted by $\mathrm{P} 1$ and $\mathrm{P} 2$, are of identical radius $a=0.5$ $\mathrm{m}$. They are seated on the $x$ axis and are placed symmetrically with respect to the $y$ axis. The net distance between the two piles ' $d_{N e t}$ ' equals three times the pile radius $a$. Other parameters hold the same magnitudes as listed in Table 2.

It is anticipated that the relative orientation between incident wave directions and the spatial arrangement of pile foundations significantly affects the behaviour of pile foundations. Thus, three representative cases are investigated in this study, corresponding to situations in which the incident wave direction is in alignment with $(\alpha=0)$, oblique with ( $\alpha=\pi / 4$ ), and orthogonal to $(\alpha=\pi / 2)$ the connecting line of the two piles, as shown in Fig. 13. Parametric analyses in terms of the incident wave number $k$ and the ratio of the pile 
Fig. 10 Time-dependent behaviour of the monopile foundation a displacement versus time for representative locations along $L-L^{\prime}$ and $\mathbf{b}$ monopile motion description for one period

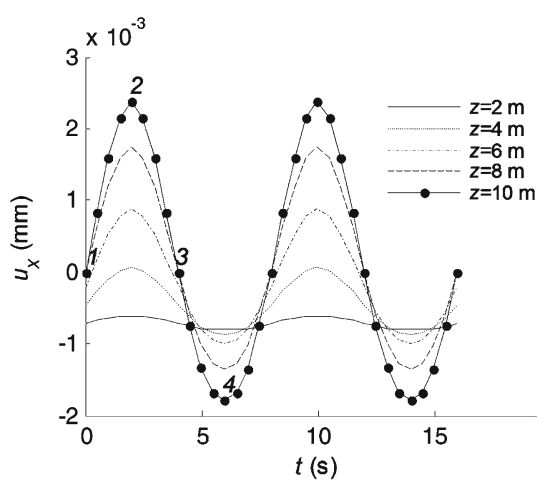

(a)

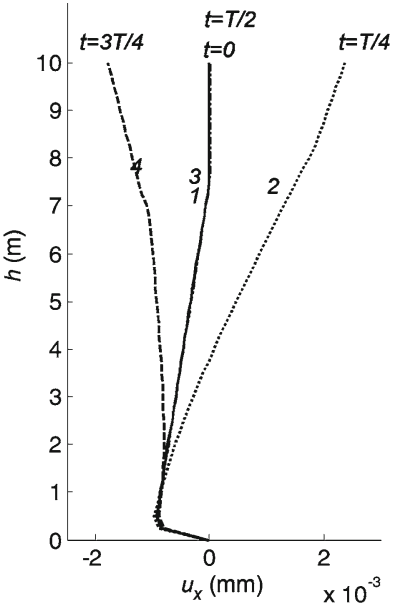

(b)

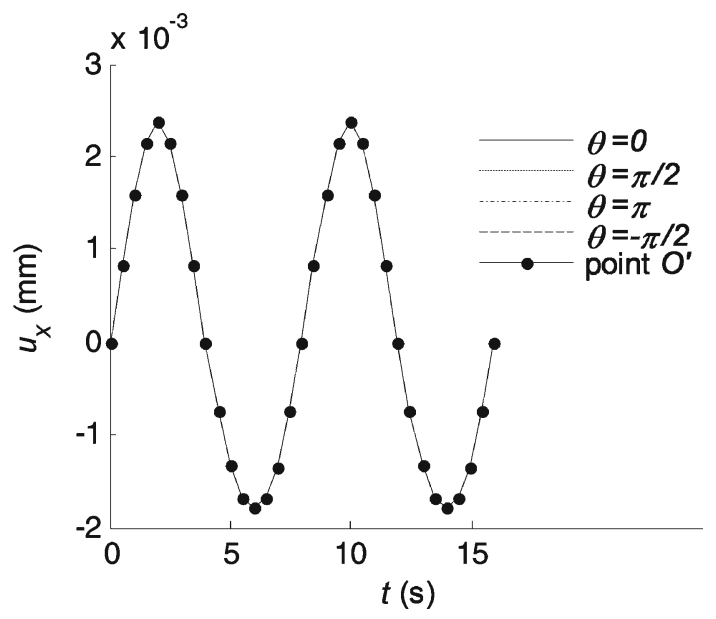

Fig. 11 Displacement versus time for representative locations on the monopile head

distance $d_{N e t}$ to the pile radius $a$, abbreviated as the dimension ratio in the following text for brevity and denoted as $e$, are concurrently carried out to present the time-dependent responses of pile foundations with respect to changing wave conditions. From relevant wave information and the dispersion equation, the wave number $k$ is calculated to change from 0.05 to $0.15 \mathrm{~m}^{-1}$ by an increment of $0.025 \mathrm{~m}^{-1}$. The dimension ratio $e$ is arranged to vary from 1 to 5 with an increment of 1 . Table 3 lists the variation of the three parameters, which will be employed in the subsequent discussions. Moreover, the influence of the variation of one parameter on the influence of other parameters on the transient response of pile foundations is also worth exploring.

\subsection{Incident wave angle $\alpha=0$}

The incident wave angle $\alpha=0$ leads to symmetrical behaviour of pile foundations with respect to the $x$ axis, and accordingly zero displacement components in the $y$ direction. Therefore, only the displacements in the $x$ direction are plotted in Fig. 14 for both piles. The displacement-versustime relationships are presented for varying $e$ (from 1 to 5) when the wave number $k=0.10 \mathrm{~m}^{-1}$, as a representative case. It is noticed that P1 and P2 have different responses to wave loads when the distance between the two piles varies.
Fig. 12 Geometric model of a group of two piles in ocean environment: a $x z$ plane view and $\mathbf{b} x y$ plane view

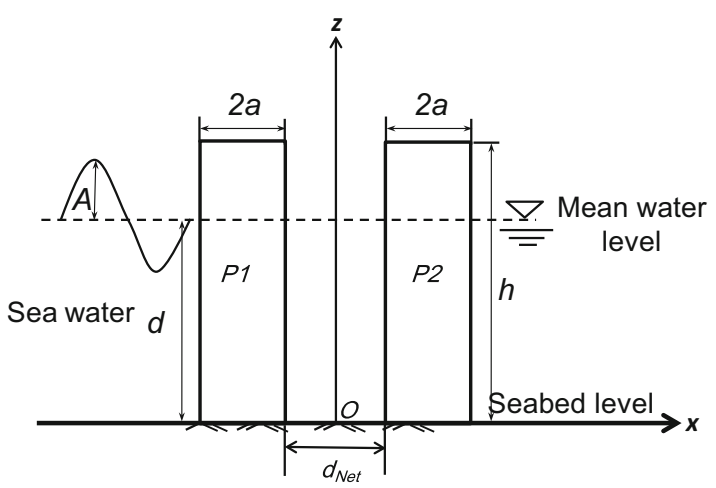

(b) 


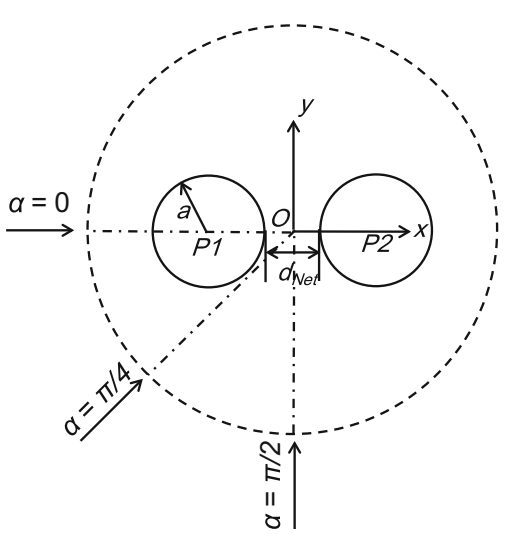

Fig. 13 Variation of the incident wave direction

Generally, for identical $e$, the displacement of $\mathrm{P} 1$ is comparatively greater than that of $\mathrm{P} 2$ by an order of magnitude 1 . For P1, the maximum displacement amplitude in the positive $x$ direction is $0.97 \times 10^{-2} \mathrm{~mm}, 0.87 \times 10^{-2} \mathrm{~mm}, 0.80 \times$ $10^{-2} \mathrm{~mm}, 0.75 \times 10^{-2} \mathrm{~mm}$ and $0.70 \times 10^{-2} \mathrm{~mm}$ when $e$ increases from 1 to 5 . At the same time instant, the displacement for $\mathrm{P} 2$ is read as $-0.18 \times 10^{-2} \mathrm{~mm},-0.313 \times 10^{-3} \mathrm{~mm}$, $0.477 \times 10^{-3} \mathrm{~mm}, 0.965 \times 10^{-3} \mathrm{~mm}$ and $0.13 \times 10^{-2} \mathrm{~mm}$, with the negative sign indicating the negative $x$ direction.

It can be further read from Fig. 14 that, with $e$ no more than 3, P1 and P2 move in the opposite directions, i.e. the motion of $\mathrm{P} 2$ has a phase difference of $T / 2$ to that of $\mathrm{P} 1$. This can be elaborated using Fig. 10b, when P1 follows the trajectory of $1 \rightarrow 2 \rightarrow 3 \rightarrow 4 \rightarrow 1, \mathrm{P} 2$ moves in accordance with $1 \rightarrow 4 \rightarrow 3 \rightarrow 2 \rightarrow 1$. This is related to the spatial proximity between the two piles. When the pile distance is relatively small, the resultant force acting on $\mathrm{P} 2$ is in the opposite direction to that on P1, and the two piles are moving one moment towards each other and the next moment away from each other. As the net distance becomes larger, the effect of P1's presence on the behaviour of the waves around P2 is not as significant, and the resultant force acting on $\mathrm{P} 2$ is in the same direction as that on P1. Consequently, both piles move in the same direction.

Figure 15 is used to explore the variation of the timedependent response of the two piles with respect to $k$ when $e=3$ as a representative case. Apparently, both piles maintain the same movement period as the external waves, which changes from $14.99,10.26,7.96,6.63$ to $5.76 \mathrm{~s}$ as the wave number varies from 0.05 to $0.15 \mathrm{~m}^{-1}$. Once again, the displacement amplitude of P1 is greater than that of $\mathrm{P} 2$ at the same time instant. The displacement amplitude is read as $0.15 \times 10^{-2} \mathrm{~mm}, 0.39 \times 10^{-2} \mathrm{~mm}, 0.80 \times 10^{-2}$ $\mathrm{mm}, 0.137 \times 10^{-1} \mathrm{~mm}$, and $0.210 \times 10^{-1} \mathrm{~mm}$ for P1 and $-0.296 \times 10^{-3} \mathrm{~mm},-0.173 \times 10^{-3} \mathrm{~mm}, 0.477 \times 10^{-3} \mathrm{~mm}$, $0.18 \times 10^{-2} \mathrm{~mm}$ and $0.41 \times 10^{-2} \mathrm{~mm}$ for $\mathrm{P} 2$ as $k$ increases from $0.05 \mathrm{~m}^{-1}$ to $0.15 \mathrm{~m}^{-1}$.

It should be noted that the time-dependent behaviour of the piles is not solely related to either $e$ or $k$. On the contrary, the piles have different performance for different combinations of $e$ and $k$. More specifically, for any $k$ not equal to 0.10 $\mathrm{m}^{-1}$, the displacement-versus-time relationships for varying $e$ would be dissimilar from Fig. 14. Analogously, when $e$ is not equal to 3 , the patterns of the transient responses of the piles with varying wave numbers would be different from Fig. 15. To provide a clear overview of this phenomenon, the variation of the maximum displacement of both piles with respect to the two parameters $e$ and $k$ is plotted in Fig. 16. It is found that: (1) the displacement amplitude of $\mathrm{P} 1$ is greater by an order of 1 in comparison with that of $\mathrm{P} 2$ for same cases of parametric combinations; (2) the displacement amplitude decreases with $e$ for any fixed $k$ for $\mathrm{P} 1$. As for $\mathrm{P} 2$, by reading the lower part of Fig. 16b, it is seen that in situations with small wave numbers or when the two piles are closely located to each other, P2 has an opposite movement direction to that of P1. In addition, the displacement mag-
Table 3 Parameter variation for parametric analysis

Fig. 14 Displacement versus time when $k=0.10 \mathrm{~m}^{1}$ and $\alpha=0$ for varying $e: \mathbf{a} \mathrm{P} 1$ and $\mathbf{b}$ $\mathrm{P} 2$

\begin{tabular}{llllll}
\hline Parameters & Magnitudes & & \\
\hline Wave number $k\left(\mathrm{~m}^{-1}\right)$ & 0.05 & 0.075 & 0.10 & 0.125 & 0.15 \\
Dimension ratio $e=d_{N e t} / a$ & 1 & 2 & 3 & 4 & 5 \\
Incident wave direction $\alpha(\mathrm{rad})$ & 0 & $\pi / 4$ & $\pi / 2$ & - & -
\end{tabular}

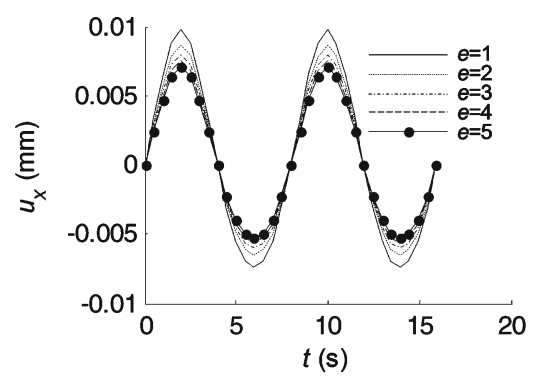

(a)

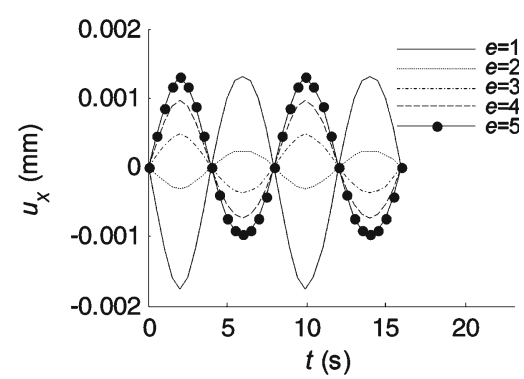

(b) 
Fig. 15 Displacement versus time when $e=3$ and $\alpha=0$ for varying $k$ : $\mathbf{a} \mathrm{P} 1$ and $\mathbf{b} \mathrm{P} 2$

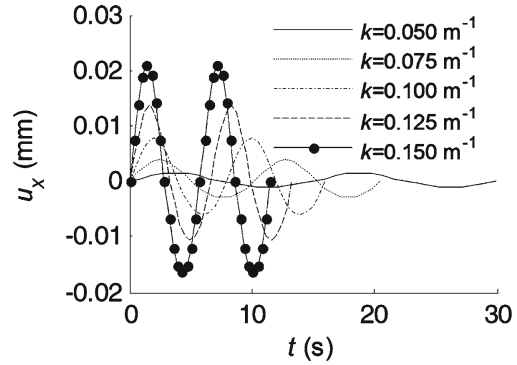

(a)

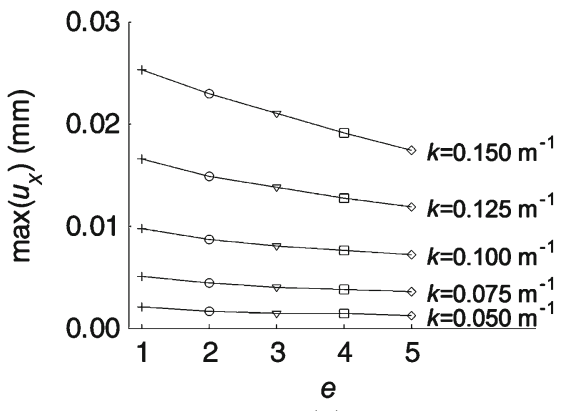

(a)

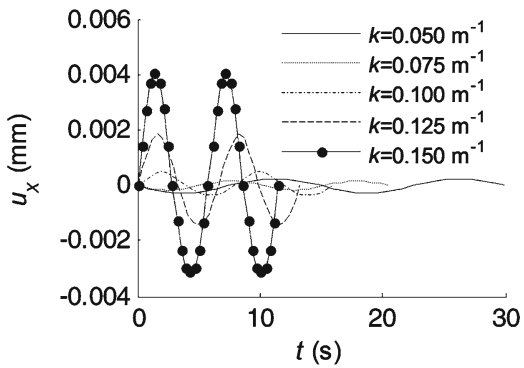

(b)

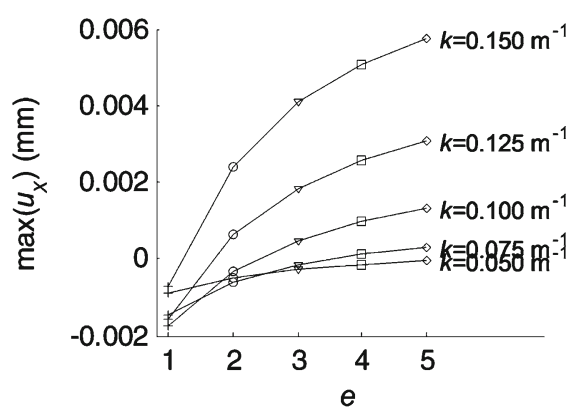

(b) nitude of $\mathrm{P} 2$ decreases as the value of $e$ increases. On the other hand, the upper part of Fig. 16b shows an increasing displacement amplitude with respect to an increasing $e$; (3) viewing any particular symbol to examine how the displacement amplitude changes with respect to $k$ for a certain $e$, it can be concluded that the displacement of P1 shows a consistent increase with $k$. As far as $\mathrm{P} 2$ is concerned, the displacement amplitude first rises and later falls with increasing $k$ for rather small $e$. For relatively large $e$, it increases steadily when the value of $k$ becomes larger. Whether the displacement amplitude is increasing or decreasing when $e$ varies, both subplots demonstrate a rather moderate slope of variation for small wave numbers, and relatively prominent variation for large wave numbers.

It would be practically meaningful to identify how the parameter combinations affect motion directions of the two piles. By examining Fig. 16b, and employing spline interpolation, it is found that the zone under the solid line in Fig. 17 signifies, for $\alpha=0$, opposite motion directions between the two piles, and vice versa, the zone above the solid line implies the same motion directions. Bearing this knowledge in mind, particular attention should be paid not only to the pile foundations, but also their supported superstructures in practical design and construction.

\subsection{Incident wave angle $\alpha=\pi / 4$}

When the incident wave direction is oblique, at an angle of $\pi / 4$, to the connecting line between the two piles, the pile's behaviour is no longer symmetric. Displacements in both $x$ and $y$ directions are examined. Figure 18 offers general

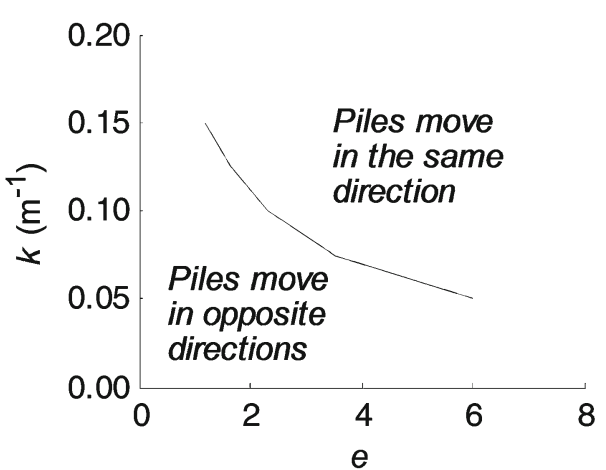

Fig. 17 Parameter combinations for pile motion directions for $\alpha=0$

knowledge as to how piles' transient behaviour vary with $e$ for $k=0.10 \mathrm{~m}^{-1}$. Calculating the resultant displacement amplitudes from the $x$ and $y$ displacement components, it is found that for $\alpha=\pi / 4$ and $k=0.10 \mathrm{~m}^{-1}$, the maximum displacement of $\mathrm{P} 1$ decreases from $0.15 \times 10^{-1} \mathrm{~mm}, 0.13$ $\times 10^{-1} \mathrm{~mm}, 0.12 \times 10^{-1} \mathrm{~mm}, 0.11 \times 10^{-1} \mathrm{~mm}$ to $0.98 \times 10^{-2}$ $\mathrm{mm}$ when $e$ increases. The corresponding readings of $\mathrm{P} 2$ are $0.54 \times 10^{-2} \mathrm{~mm}, 0.38 \times 10^{-3} \mathrm{~mm}, 0.27 \times 10^{-2} \mathrm{~mm}, 0.19 \times$ $10^{-2} \mathrm{~mm}$ and $0.14 \times 10^{-2} \mathrm{~mm}$, respectively. Regardless of the direction of motion, the displacement amplitude of both piles decreases as $e$ increases.

By reading the negative displacement associated with the motion of $\mathrm{P} 2$ in Fig. 18b, d, it is derived that when $e$ is small, the resultant motion of $\mathrm{P} 2$ has a phase difference of $T / 2$ to that of $\mathrm{P} 1$; hence, the two piles show opposite directions of motion. When the net distance between the two piles is large enough $(e \geq 4)$, they move harmoniously with the same phase. 
Fig. 18 Displacement versus time when $k=0.10 \mathrm{~m}^{-1}$ and $\alpha=\pi / 4$ for varying $e$ : $\mathbf{a} u_{x}$ of $\mathrm{P} 1 ; \mathbf{b} u_{x}$ of $\mathrm{P} 2 ; \mathbf{c} u_{y}$ of $\mathrm{P} 1$ and $\mathbf{d}$ $u_{y}$ of $\mathrm{P} 2$
Fig. 19 Displacement versus time when $e=3$ and $\alpha=\pi / 4$ for varying $k$ : $\mathbf{a} u_{x}$ of $\mathrm{P} 1 ; \mathbf{b} u_{x}$ of P2; c $u_{y}$ of P1 and $\mathbf{d} u_{y}$ of P2

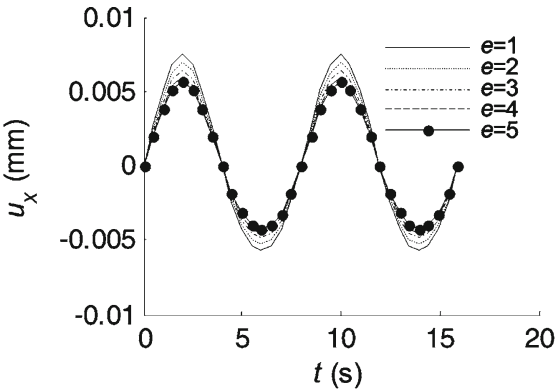

(a)

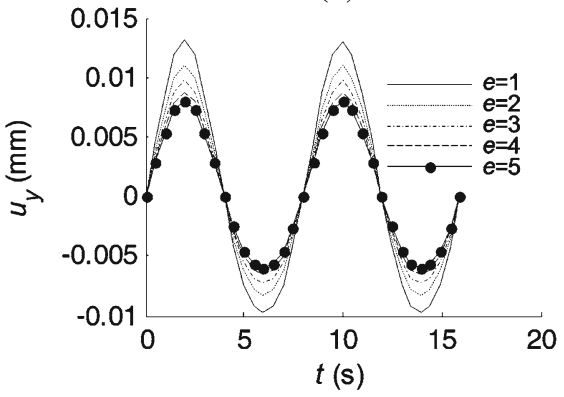

(c)

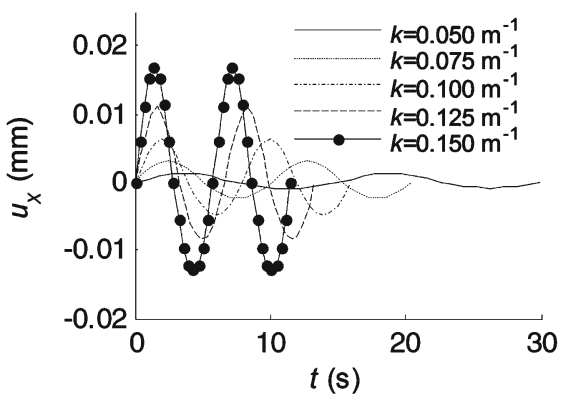

(a)

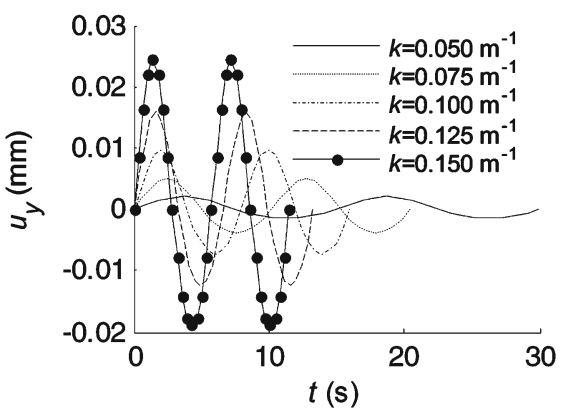

(c)

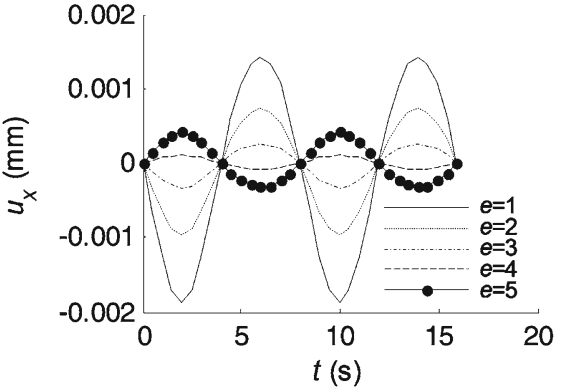

(b)

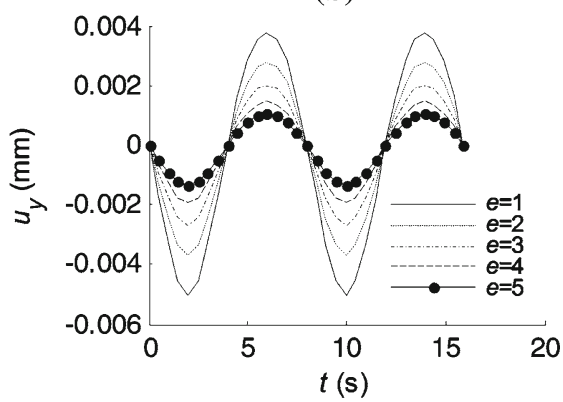

(d)

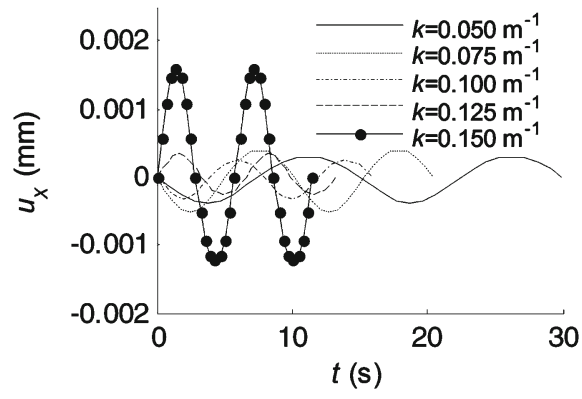

(b)

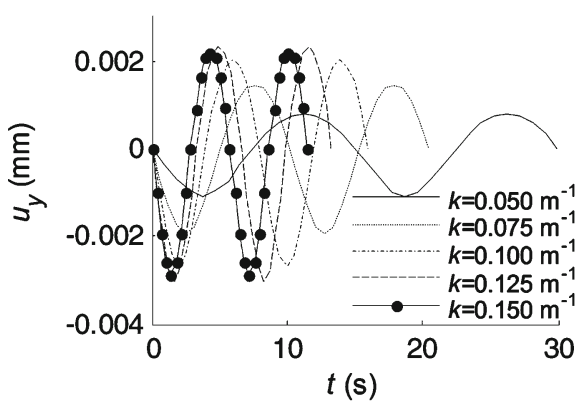

(d)
Figure 19 suggests the fluctuation of the displacement amplitude with respect to time for various $k$ when $e=3$. For $\mathrm{P} 1$, the resultant displacement consistently increases from $0.24 \times 10^{-2} \mathrm{~mm}, 0.60 \times 10^{-2} \mathrm{~mm}, 0.116 \times 10^{-1} \mathrm{~mm}, 0.20$ $\times 10^{-1} \mathrm{~mm}$ to $0.30 \times 10^{-1} \mathrm{~mm}$ as the value of $k$ increases. The resultant response of P2, calculated from Fig. 19b, d, presents a value of $0.12 \times 10^{-2} \mathrm{~mm}, 0.20 \times 10^{-2} \mathrm{~mm}$, $0.27 \times 10^{-2} \mathrm{~mm}, 0.31 \times 10^{-2} \mathrm{~mm}$ and $0.33 \times 10^{-2} \mathrm{~mm}$ for $k=0.05 \mathrm{~m}^{-1}, 0.075 \mathrm{~m}^{-1}, 0.10 \mathrm{~m}^{-1}, 0.125 \mathrm{~m}^{-1}$ and
$0.15 \mathrm{~m}^{-1}$, respectively. It is further observed that, whatever $k$ is when $e=3$ and $\alpha=\pi / 4$, the direction of motion of $\mathrm{P} 2$ is neither the same as nor opposite to that of P1, but with a certain angle between them.

Figure 20 is drawn to assist the understanding of the variation of the maximum displacement with respect to the two parameters, $e$ and $k$, when $\alpha=\pi / 4$. P1 presents a rather simple reduction in the amplitude as $e$ increases for each individual $k$, as seen in Fig. 20a, c. The reduction is more obvious 
Fig. 20 Displacement versus $e$ for varying $k$ when $\alpha=\pi / 4$ for: a maximum $u_{x}$ for $\mathrm{P} 1$; $\mathbf{b}$ maximum $u_{x}$ for $\mathrm{P} 2$; $\mathbf{c}$ maximum $u_{y}$ for $\mathrm{P} 1$ and $\mathbf{d}$ maximum $u_{y}$ for $\mathrm{P} 2$

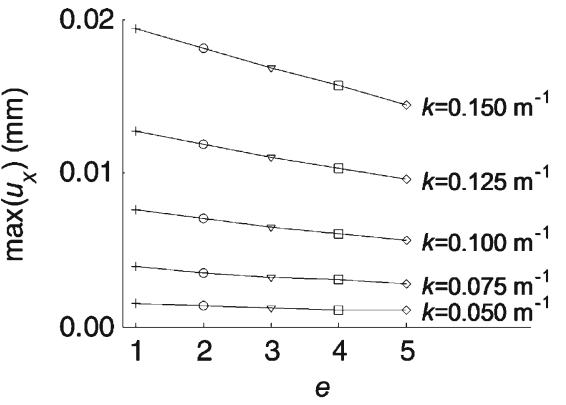

(a)

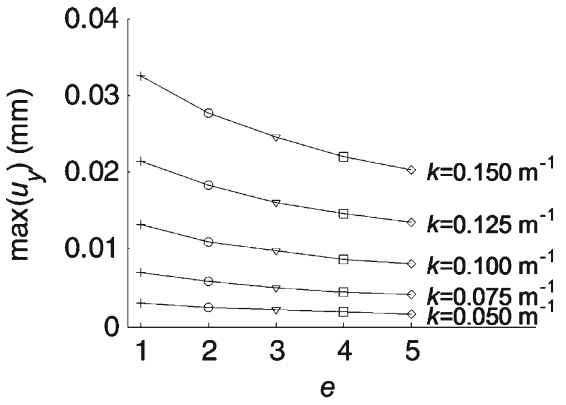

(c)

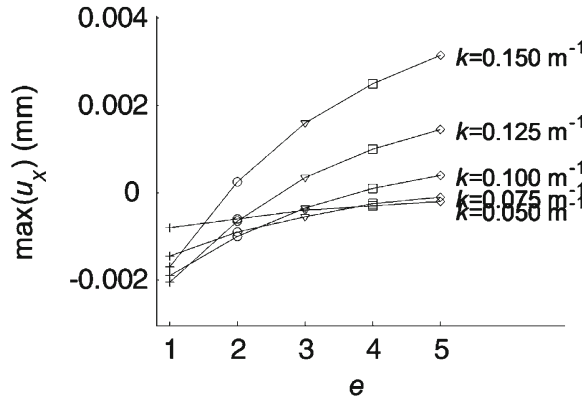

(b)

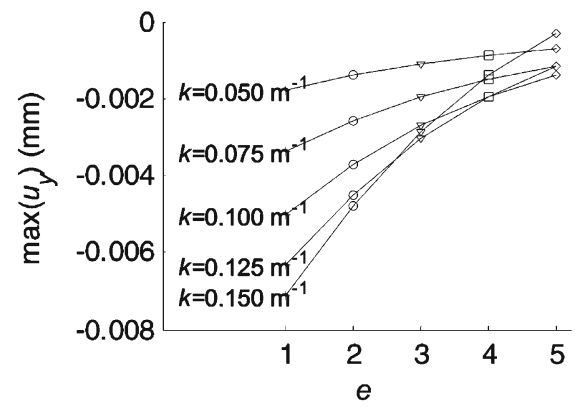

(d) when the wave number is greater. The amplitude increases with $k$ for any specific $e$, which is evident by viewing the same legend markers in Fig. 20a, c, and the increase becomes more remarkable as $k$ becomes greater. The displacement amplitude of P2, first of all, is comparatively smaller than its counterpart P1 by roughly an order of 1 . It is calculated from the $x$ and $y$ displacement components, shown respectively in Fig. 20b, d, that the resultant displacement decreases with $e$ for any specific $k$, and the decrease becomes more significant when $k$ is large. By fixing $e$ and examining the variation of the displacement amplitude with respect to $k$, it is shown that the resultant displacement monotonically increases with $k$ and the increase becomes less significant as the value of $k$ rises.

Overall, both piles show relatively greater displacement with large wave numbers. When the two piles are closely spaced or when the wave number is small, they tend to have phase difference and move towards different directions. It is found that the direction of motion of P1 is roughly in alignment with the incident wave direction, with an angle varying between $\pi / 4$ and $\pi / 3$. This is slightly greater than the incident wave angle due to the presence of P2. As far as P2 is concerned, the parameter combination has a significant influence on the direction of motion of P2. As illustrated in Fig. 21a, for the $e$ and $k$ combinations defined above the solid line, the range of the direction of motion of $\mathrm{P} 2$ is $[-\pi / 20]$, as shown in Fig. 21b. It shifts to $[-2 \pi / 3-\pi / 2]$ when $e$ and $k$ are confined in the region below the solid line. The complex variation feature of the displacement of P2 once again proves that both wave properties and the spatial layout of pile members affect the pile group's response, which should be

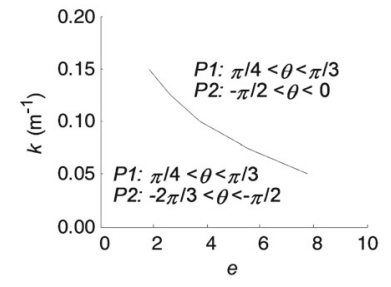

(a)

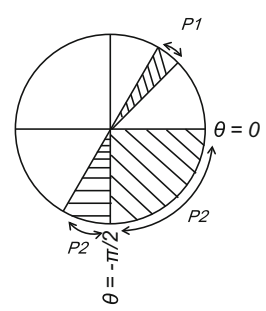

(b)
Fig. 21 Illustration of pile motion directions for $\alpha=\pi / 4$ : a parameter combinations and $\mathbf{b}$ polar coordinate representation

taken into consideration in the foundation design and safety evaluation processes.

\subsection{Incident wave angle $\alpha=\pi / 2$}

When the incident wave direction is orthogonal to the connecting line between the two piles, the physical configuration is symmetrical with respect to the $y$ axis. Therefore, both piles exhibit the same displacement amplitude but opposite motion directions along the $x$ axis. In the $y$ direction, they hold identical motion pattern both in magnitudes and directions. The resultant displacement amplitudes thus are identical for both piles, with magnitudes of $0.68 \times 10^{-2} \mathrm{~mm}$, $0.62 \times 10^{-2} \mathrm{~mm}, 0.58 \times 10^{-2} \mathrm{~mm}, 0.56 \times 10^{-2} \mathrm{~mm}$ and $0.54 \times 10^{-2} \mathrm{~mm}$ for increasing $e$ when $k=0.10 \mathrm{~m}^{-1}$ and $\alpha=\pi / 2$. This is demonstrated in Fig. 22, which shows the displacement components of both piles for varying $e$. 
Fig. 22 Displacement versus time when $k=0.10 \mathrm{~m}^{1}$ and $\alpha=\pi / 2$ for varying $e: \mathbf{a} u_{x}$ of $\mathrm{P} 1 ; \mathbf{b} u_{x}$ of $\mathrm{P} 2 ; \mathbf{c} u_{y}$ of $\mathrm{P} 1$ and $\mathbf{d}$ $u_{y}$ of $\mathrm{P} 2$
Fig. 23 Displacement versus time when $e=3$ and $\alpha=\pi / 2$ for P1 for varying $k$ : $\mathbf{a}$ in the $x$ direction and $\mathbf{b}$ in the $y$ direction

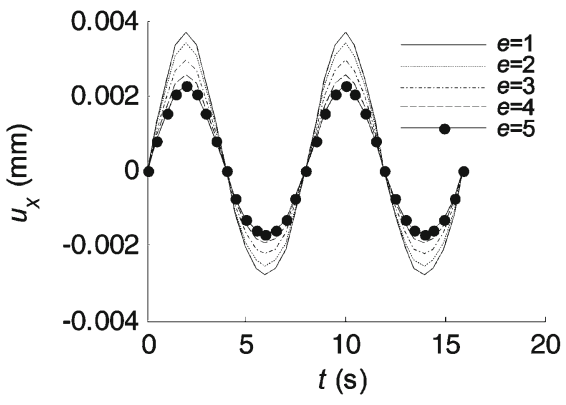

(a)

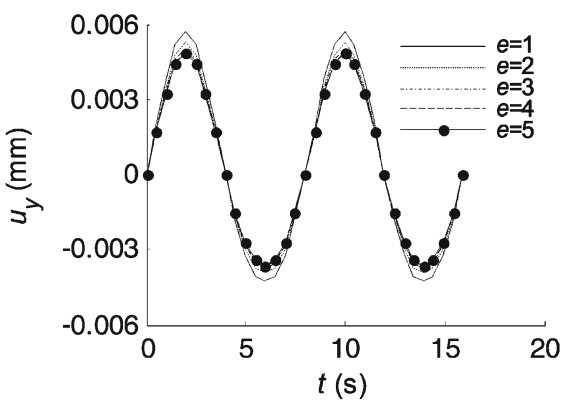

(c)

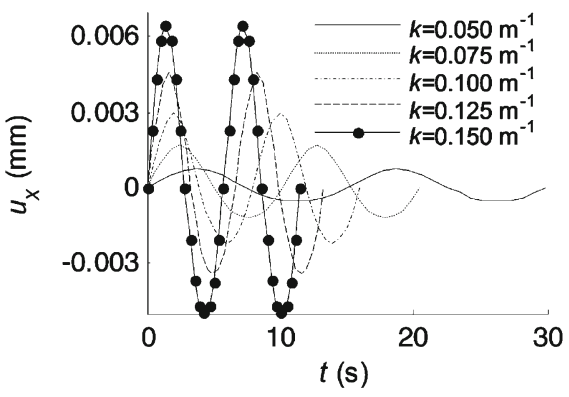

(a)

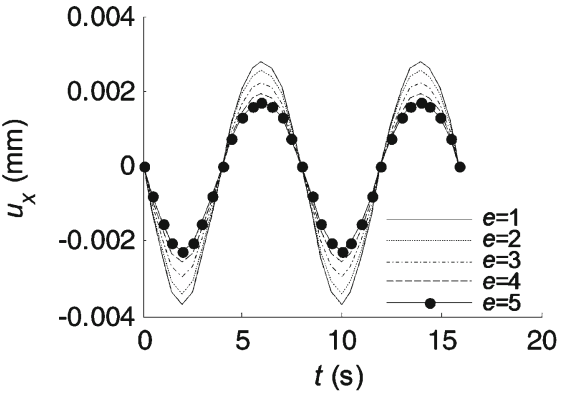

(b)

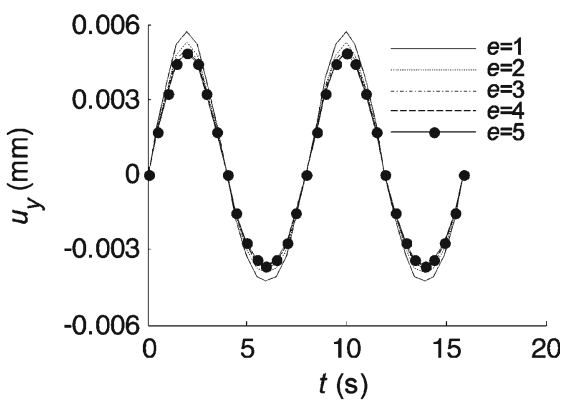

(d)

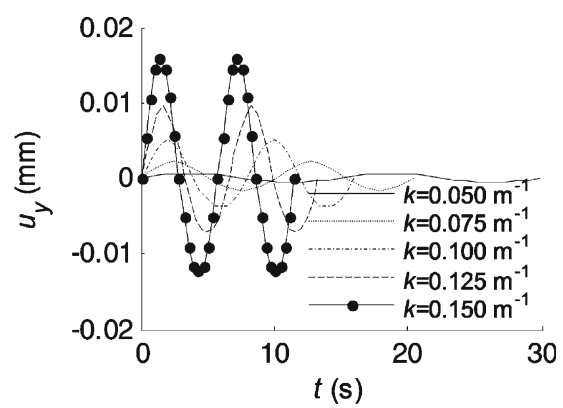

(b)
Figure 23 depicts the displacement history in both $x$ and $y$ directions of $\mathrm{P} 1$ for varying $k$ when $e=3$. The resultant displacement amplitudes are calculated as $0.10 \times 10^{-2} \mathrm{~mm}$, $0.27 \times 10^{-2} \mathrm{~mm}, 0.58 \times 10^{-2} \mathrm{~mm}, 0.11 \times 10^{-1} \mathrm{~mm}$ and $0.17 \times 10^{-1} \mathrm{~mm}$ for every increasing $k$. The corresponding plots for $\mathrm{P} 2$ should present identical features of the displacement variation in the $y$ direction, but opposite directions of motion in the $x$ direction.

Figure 24 shows the variation of displacement amplitude with respect to $e$ for varying $k$ for P1 only. Both the $x$ and $y$ displacement components, and therefore the resultant placement amplitude, decrease when $e$ increases from 1 to 5 , and the decrease is more distinct when the wave number is greater. In addition, it is evident that the displacement amplitude increases with $k$, and the smaller the net distance between the two piles, the quicker the displacement amplitude increases. In terms of the motion directions, due to the symmetry with respect to the $y$ axis associated with the problem configuration when $\alpha=\pi / 2$, it is found that the resultant motion directions of the two piles are always symmetric with respect to the incident wave direction. Under the specified parametric variations considered in this study, the direction of motion of $\mathrm{P} 1$ lies in $\pi / 6<\theta<5 \pi / 12$, with the corresponding direction of $\mathrm{P} 2$ being $5 \pi / 6>\theta>7 \pi / 12$, and no phase difference is identified.

\section{Conclusions}

This study investigates the time-dependent behaviour of pile foundations using SBFEM when subjected to ocean wave loads. A modal analysis reveals that pile foundations examined in this study have significantly greater natural frequencies than those of external waves. Therefore, resonance will rarely happen for the specified situation. Considering the material damping effect, it is found that individual pile foundations arrive at a steady-state vibration shortly after the action of external wave pressures. Piles move with the same 
Fig. 24 Displacement versus $e$ of $\mathrm{P} 1$ for varying $k$ when $\alpha=\pi / 2$ : $\mathbf{a}$ in the $x$ direction and $\mathbf{b}$ in the $y$ direction

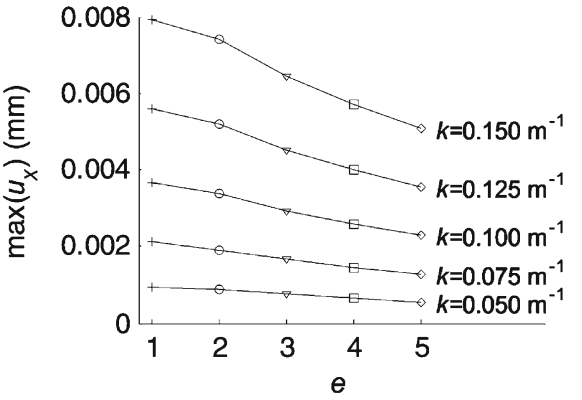

(a)

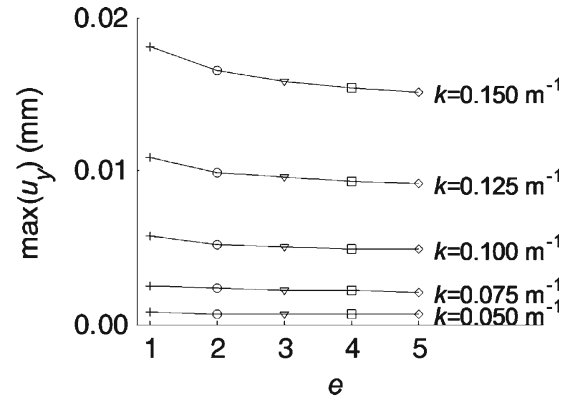

(b) period as waves, and the bigger the wave number, the shorter the period. For pile group foundations, especially the group of two piles considered in this study, the responses of piles are significantly affected by the problem configuration in terms of the spatial layout of pile members and the relative wave properties. Emphasis is placed on two predominant parameters: the wave number $k$ and the ratio $e$ of the pile distance to the pile radius. The displacement amplitude of the leading pile presents a rather moderate decrease with $e$ when $k$ is small, but the decrease is quite significant when the value of $k$ is large. The displacement increases with $k$ for any constant $e$, and the increase is slightly more obvious in cases with a lower $e$ than that with a higher one. The succeeding pile always presents a smaller displacement amplitude than that of the leading pile. It displaces mildly as the value of $e$ changes for small wave numbers and shows substantial response when the wave number is large. The discussions on the relative motion directions of the two piles show that when the wave number is small, or when the two piles are closely spaced, they tend to have phase differences and move in different directions. This should be thoroughly considered when suggesting relative locations between the pile foundations, also when designing or evaluating the safety of their supported superstructures.

This study provides a clear picture as to how individual pile foundations behave under the time-dependent wave pressure. At the same time, it provides instructive information on the transient response of pile group foundations to varying wave conditions. It is worth mentioning the SBFEM model proposed in this study is applicable for investigations of transient responses of pile foundations having any arbitrary number of piles of various cross-sections and spatial layouts. To guide engineering design when a specific project is involved, external loads from wind actions acting on wind farm monopiles or superstructures acting on pile groups need to be applied in the SBFEM model, following the same way as in other numerical techniques (FEM for example). Furthermore, the effects of the unbounded seabed can be addressed by formulating an SBFEM solution for the Biot's equation (Jeng and Zhang 2005; Zhang and Jeng 2005). This can be incorporated in the presented model as a continuation of the current study. The derivation and validation of the formulation are in progress.

\section{References}

Bai W, Taylor RE (2006) Higher-order boundary element simulation of fully nonlinear wave radiation by oscillating vertical cylinders. Appl Ocean Res 28(4):247-265

Bai W, Taylor RE (2009) Fully nonlinear simulation of wave interaction with fixed and floating flared structures. Ocean Eng 36(3):223-236

Benassai G (2006) Introduction to coastal dynamics and shoreline protection. WIT Press, Southampton

Chakrabarti SK, Tam WA (1975) Interaction of waves with large vertical cylinder. J Ship Res 19:11

Chatjigeorgiou IK, Mavrakos SA (2010) An analytical approach for the solution of the hydrodynamic diffraction by arrays of elliptical cylinders. Appl Ocean Res 32(2):242-251

Chen J-T, Lee Y-T, Lin Y-J (2009) Interaction of water waves with vertical cylinders using null-field integral equations. Appl Ocean Res 31(2):101-110

Dean RG, Dalrymple RA (1991) Water wave mechanics for engineers and scientists. World Scientific, Singapore

Ducrozet G, Bingham HB, Engsig-Karup AP, Ferrant P (2010) Highorder finite difference solution for 3D nonlinear wave-structure interaction. J Hydrodyn Ser B 22(5, Supplement 1):225-230

Feng B, Zheng YH, You YG, Wu BJ, Sheng SW (2008) Interaction of linear waves with an array of infinitely long horizontal circular cylinders in a two-layer fluid of infinite depth. Ocean Eng 35(10):963-982

Finnegan W, Goggins J (2012) Numerical simulation of linear water waves and wave-structure interaction. Ocean Eng 43:23-31

Ge F, Lu W, Wu X, Hong Y (2010) Fluid-structure interaction of submerged floating tunnel in wave field. Procedia Eng 4:263-271

Gōda Y (2010) Random seas and design of maritime structures, 3rd edn. World Scientific, Singapore

Huang JB, Taylor RE (1996) Semi-analytical solution for second-order wave diffraction by a truncated circular cylinder in monochromatic waves. J Fluid Mech 319:171-196

Jeng DS, Ye JH, Zhang JS, Liu PLF (2013) An integrated model for the wave-induced seabed response around marine structures: model verifications and applications. Coastal Eng 72:1-19

Jeng DS, Zhang H (2005) An integrated three-dimensional model of wave-induced pore pressure and effective stresses in a porous seabed: II. Breaking waves. Ocean Eng 32(16):1950-1967

Jung KH, Chang K-A, Huang ET (2004) Two-dimensional flow characteristics of wave interactions with a fixed rectangular structure. Ocean Eng 31(8-9):975-998 
Lee HH, Wang PW (2000) Dynamic behavior of tension-leg platform with net-cage system subjected to wave forces. Ocean Eng 28(2):179-200

Li B (2007) Extending the scaled boundary finite element method to wave diffraction problems. The University of Western Australia, Crawley

Li M, Guan H, Zhang H, Liu J (2013a) Three-dimensional investigation of wave-pile group interaction using the scaled boundary finite element method-Part II: application results. Ocean Eng 64:185195

Li M, Song H, Zhang H, Guan H (2010) Structural response of offshore monopile foundations to ocean wave loads. In: Proceedings of the 9th ISOPE Pacific/Asia offshore mechanics symposium (ISOPE PACOMS-2010), Busan, Korea, pp 66-73

Li M, Zhang H, Guan H (2011) Study of offshore monopile behaviour due to ocean waves. Ocean Eng 38(17-18):1946-1956

Li M, Zhang H, Guan H, Lin G (2013b) Three-dimensional investigation of wave-pile group interaction using the scaled boundary finite element method. Part I: theoretical developments. Ocean Eng 64:174-184

Li M, Zhang Y, Zhang H, Guan H (2015) Numerical stability and accuracy of the scaled boundary finite element method in engineering applications. ANZIAM J 57(02):114-137

Liu X, Xu H, Shao S, Lin P (2013) An improved incompressible SPH model for simulation of wave-structure interaction. Comput Fluids 71:113-123

Malenica Š, Taylor RE, Huang J (1999) Second-order water wave diffraction by an array of vertical cylinders. J Fluid Mech 390:349 373

Marburg S (2002) Six boundary elements per wavelength: is that enough? J Comput Acoust 10(01):25-51

Mei CC (1989) The applied dynamics of ocean surface waves. World Scientific, Singapore

Moan T (2005) Safety of offshore structures. Center for Offshore Research and Engineering, National University of Singapore, Singapore, Report (2005-04)

Mostafa YE, El Naggar MH (2004) Response of fixed offshore platforms to wave and current loading including soil-structure interaction. Soil Dyn Earthq Eng 24(4):357-368

Phillips OM (1977) The dynamics of the upper ocean, 2nd edn. Cambridge University Press, Cambridge

Sommerfeld A (1949) Partial differential equations in physics. Academic Press, New York

Song C (2009) The scaled boundary finite element method in structural dynamics. Int J Numer Methods Eng 77(8):1139-1171

Song H, Tao L, Chakrabarti S (2010) Modelling of water wave interaction with multiple cylinders of arbitrary shape. J Comput Phys 229(5):1498-1513

Song H, Tao LB (2009) Hydroelastic response of a circular plate in waves using scaled boundary FEM. Omae 1:247-254

Spring BH, Monkmeyer PL (1974) Interaction of plane waves with vertical cylinders. In: Proceedings of the 14th international conference on coastal engineering, vol. 1 , no 14

Srisupattarawanit T, Niekamp R, Matthies HG (2006) Simulation of nonlinear random finite depth waves coupled with an elastic structure. Comput Methods Appl Mech Eng 195(23-24):3072-3086

Tao L, Song H (2008) Solving water wave diffraction by an elliptic cylinder using scaled boundary finite element method. Aust N Z Ind Appl Math J 50:C474-C489
Tao L, Song H, Chakrabarti S (2007) Scaled boundary FEM solution of short-crested wave diffraction by a vertical cylinder. Comput Methods Appl Mech Eng 197(1-4):232-242

Tao L, Song H, Chakrabarti S (2009a) Scaled boundary FEM model for interaction of short-crested waves with a concentric porous cylindrical structure. J Waterway Port Coast Ocean Eng 135(5):200212

Tao L, Song H, Chakrabarti S (2009b) Spacing effect on hydrodynamics of two adjacent offshore caissons. In: ASME Conference Proceedings 2009, no 43444, pp 127-133

United States Coast Guard (1984) Marine casualty report: mobile offshore drilling unit (MODU) ocean ranger, O.N. 615641, capsizing and sinking in the atlantic ocean on 15 February 1982 with multiple loss of life, USCG 0001 HQS 82. The Guard, Washington DC

Wang C, Wu G (2007) Time domain analysis of second-order wave diffraction by an array of vertical cylinders. J Fluids Struct 23(4):605-631

Wang CZ, Wu GX (2010) Interactions between fully nonlinear water waves and cylinder arrays in a wave tank. Ocean Eng 37(4):400 417

Wheeler JD (1969) Methods for calculating forces produced by irregular waves, offshore technology conference. In: Offshore Technology Conference, Houston, United States of America

Wolf JP, Song CM (1996) Finite-element modelling of unbounded media. Wiley, Chichester

Wu C, Watanabe E, Utsunomiya T (1995) An eigenfunction expansionmatching method for analyzing the wave-induced responses of an elastic floating plate. Appl Ocean Res 17(5):301-310

Wu CS, Young DL, Chiu CL (2013) Simulation of wave-structure interaction by hybrid Cartesian/immersed boundary and arbitrary Lagrangian-Eulerian finite-element method. J Comput Phys 254:155-183

Ye J, Jeng D, Liu PLF, Chan AHC, Wang R, Zhu C (2014) Breaking wave-induced response of composite breakwater and liquefaction in seabed foundation. Coastal Eng 85:72-86

Ye J, Jeng D, Wang R, Zhu C (2013a) A 3-D semi-coupled numerical model for fluid-structures-seabed-interaction (FSSI-CAS 3D): model and verification. J Fluids Struct 40:148-162

Ye J, Jeng D, Wang R, Zhu C (2013b) Validation of a 2-D semi-coupled numerical model for fluid-structure-seabed interaction. J Fluids Struct 42:333-357

Ye J, Jeng D, Wang R, Zhu C (2015) Numerical simulation of the wave-induced dynamic response of poro-elastoplastic seabed foundations and a composite breakwater. Appl Math Model 39(1):322-347

Zhang H, Jeng D-S (2005) An integrated three-dimensional model for wave-induced seabed response in a porous seabed. I. A sloping seabed. Ocean Eng 32(5):701-729

Zhong Z, Wang KH (2009) Modeling fully nonlinear shallow-water waves and their interactions with cylindrical structures. Comput Fluids 38(5):1018-1025

Zhu S (1993) Diffraction of short-crested waves around a circular cylinder. Ocean Eng 20(4):389-407 